\title{
The Experimental Flesh: Incarnation in Terms of Quantum Measurement and Phenomenological Perception
}

Will Johncock, University of New South Wales (Sydney, Australia)

Email: z3137140@zmail.unsw.edu.au

\section{Abstract}

What is the relation of the human to the world and the things in it? Do the various forms of human interrogation of the world discover things, and with them, a world? That is, can we reduce Being to a separation of knower from what can be known, or of observer from what can be observed? This article interrogates the question of the human-world relation via an interdisciplinary analysis. The "flesh of the world" phenomenology of Maurice Merleau-Ponty problematises the assumption that there is an inherent distinction between subject and object, by instead identifying the incarnation of both in the embodied act of perception. Merleau-Ponty's philosophy of a reality-as-flesh will be employed alongside, and inside, physicist Niels Bohr's demand that the constituent elements of physical reality emerge via the concurrent incarnation of the measurer and the measured during experimental procedures. Bohrian quantum physics is shown (in a manner which does not require the reader to be experienced in quantum theories) to evoke the co-manifestation of subject and object for which Merleau-Ponty argues. In blurring notions of observer and observed, an ontologically productive, rather than epistemologically interrogative, operation is identified. This is used to investigate the human-world relation, and to argue against the notion that this relation, as a condition for there being phenomena, separates knower from what can be known, or observer from what can be observed.

\section{Introduction}

What is the relation of the human to the world and the things within it? Do the various forms of human interrogation of the world discover things, and with them, a world? That is, can we reduce Being to a separation of knower from what can be known, or of observer from what can be observed? The ontological investment of this query is what is at stake in this article. In the continental philosophical tradition, the view that the natural sciences are the only, or most accurate, method of understanding phenomena is challenged. The French phenomenologist Maurice Merleau-Ponty duly argues that many appreciations of phenomena, such as, but not exclusively restricted to, those provided by the natural sciences, neglect a pre-theoretical, experiential realm that conditions the very phenomena with which they are concerned. This 
article will interrogate the broader applicability of a phenomenological interpretation of the human-world relation by first engaging the interrogation of the subject/object divide which underpins Merleau-Ponty's “flesh of the world” philosophy. This is not to suggest that an assumption of the polarisation of subject from object is exclusive to the inquiries of the natural sciences. Indeed, the intentions of this article suggest the contrary, given that it is the congruence of a phenomenological challenge to the subject/object binary, with a similar contestation provided by quantum physics, which will be our focus. In this regard, Merleau-Ponty's phenomenological appreciation of perception will be "fleshed out" via an analysis comparing it with Danish quantum physicist Bohr's explorations into the concurrent incarnation of the measurer and the measured during scientific experimentation. This Bohrian interpretation of the emergence of physical reality, when employed alongside, and inside, Merleau-Ponty's reality as flesh, will contribute to our appreciation of the ontological movement which frames the humanworld relation, a relation which conditions there being phenomena at all.

\section{Mind-Body Interaction}

When considering the human-world relation, the philosopher René Descartes, in an era associated with the Enlightenment's prioritisation of rational processes, doubted the existence of things that he perceived externally, including his own physicality. This was in part due to the potential of such perceptions to be dream-like illusions. Ironically, it was through such doubt that Descartes would find certainty of the existence of the self. For "to doubt" was "to think," and "to think" was to be a self-aware, thinking creature. Thus, as philosopher Stephen Hetherington notes, Descartes was "unable to doubt his own existence, even while accepting that he can doubt the accuracy of such beliefs as those about his body - including his having one” (2004, p. 8). However, that his now definite mind possessed a "clear and distinct" conception of his body convinced Descartes that if another aspect to the world besides one's mind existed, it could only be so in a corporeal or extended form, with which he, as a thinking creature, causally interacts. As he states, if a body is to exist, "the only alternative is that it is in another substance distinct from me” (Descartes in Cooney, 2000, p. 20).

From this came the problematic notion of explaining interaction between what is considered to be immaterial - the mind, and that which is "clearly and distinctly" material—the body. Merleau-Ponty argues that one can only draw this distinction between the mental and the physical by virtue of the existential structures involved in one's being in the world. That is, the experience of being human is necessary in order to have the concept of the mental and the concept of the physical. This primordiality for the human is characterised as an experiential mode. Merleau-Ponty duly refers to the perceptual level at which humans exist, in order to determine what the world consists of, and "what it is for it to exist" (1968, p. 96). In questioning the manner that arguments, such as that of Descartes', pre-suppose the notion of a thing in itself, something that is set in opposition to the internality of the human, Merleau-Ponty asks how is it that one's gaze reaches a thing, which appears at the end of such perception, but also occurs within the human.

\section{The Body, the Object Which does not Leave the Subject}


By employing lived experience, rather than sceptical doubt, as a foundation for this investigation, Merleau-Ponty notes the primordial, embodied relation between oneself and an object of perception, in that "the table before me sustains a singular relation with my eyes and my body" (1968, p. 7). This means that there are not two tables to every experience, that is, one in the mind and one in the world, but a single table occurring in a worldly act of perception. Because one's bodily movement, or physical positioning, alters one's perception, similarly one must acknowledge a "corporeal component" to experience (1968, p. 8). As an embodied creature, the subject is situated in a certain perspective to the world and things in it. Philosopher Elizabeth Grosz succinctly observes that this perspective determines that one's "body for Merleau-Ponty is the very condition of our access to, and conception of, space,” (1994, p. 91), whereby the body defines, and is defined by, its relation to things in the world.

This perceptual, corporeal, human-world relation contradicts conceptions of the body as being an assemblage of distinct, external parts experienced by a "disembodied knower." Such knowledge would be a reflective production, installing positive notions of before and after. Reflection, after all, "by its very nature is re-flective, it comes second and looks back at something more primary” (Merleau-Ponty, 1968, p. 34). Merleau-Ponty thus re-conceives of the body beyond its status of Cartesian-object, subservient to an immaterial mind. This problematisation of the subject/object binary is exemplified in the touching/touched body. In touching one's other hand, a subject concurrently touches and is touched, "through this crisscrossing within (the hand(s)) of the touching and the tangible” (1968, p. 133). That in any act of perception, one is never removed from their body, that object which does not leave them, thus challenges the notion that one's corporeality can ever be pure "object." The body is the thing that experiences itself, and indeed other things, from the inside of experience.

The sensible world is not comprised of a distinct subject that perceives (touches) distinct objects, but rather "touch is formed in the midst of the world and as it were in the things" (1968, p. 134). My impression of "thing" here is of its synonymy with the term "experience," in suggesting that every object in or of the world is a verb-like process of becoming. Perception is not something that provides the embodied subject with access to the world, but rather embodied perception is only possible because it is of the world, whereby "he who sees cannot possess the visible unless he is possessed by it, unless he is of it" (1968, p. 134). To see, therefore, is to possess a body that is capable of being seen. ${ }^{1}$ This dichotomy shows that the distance between the body and the world is a proximal one. The distinction of one thing from another is produced by the look and/of the body, which as flesh is "constitutive for the thing of its visibility as for the seer of his corporeity" (1968, p. 135). Bodily perception chiasmatically puts itself in contact with things in the world and separates them at the same time, as the seer and seen, as the toucher and touched, within this "fabric of experience, this flesh of time” (1968, p. 111). 


\section{No Outside of Body}

The immediacy of fleshed perception that constitutes this implicated relation with things, necessarily includes bodies-as-things, meaning that "the perception of the bodies of others is as primary as the perception of one's own body" (Schilder, 1950, p. 218). This continual interchange between their own body and that of others takes Merleau-Ponty's conception of flesh to an inevitable point of interrogation, whereby as the early $20^{\text {th }}$ century Austrian psychoanalyst Paul Schilder notes, "there now arises the question of what is our own body and what is the body of others” (1950, p. 235). Interestingly, for Schilder and Merleau-Ponty, such receptivity proposes that there is no "outside" the body. As we have seen, according to Merleau-Ponty, Being is experienced not from the "depths of nothingness," but from the "midst of itself" (Merleau-Ponty, 1968, p. 113). It follows that the nature of the body's receptivity with a world of which it is means that there is nothing in the world, which cannot be, or is not already, incorporated. Like an amorphous blob the body-image of the subject expands beyond their presumed corporeal limits. Consequently, "a stick, a hat, clothes, become part of the bodyimage” (Schilder, 1950, p. 213). Objects are not incorporated or passed on in a linear temporal fashion by the subject, in terms of what the body was before or after such objects were incorporated. Rather, such incorporations represent trans-temporal manifestations of the same self-comprehendible, plastic incarnation of that flesh which was already body.

That the body and the world flow over into one another indistinguishably means that the point where the individual body ends and the world begins is blurred. In thinking the limits of the body, one must not conceive of an inside and an outside, permeating each other. Rather than demarcating one body from another, or indeed one body from any "thing," what is instead required is a re-conceptualisation of the notion of the limit which sets the natural human in opposition to that which is, seemingly, artificially non-human. Consequently, what we can take from Merleau-Ponty's theory of body is that nature and culture are not polarised, but inhabit and produce the same embodied Being.

\section{An Involvement that is Anterior to Representationalism}

How operative this nature/culture divide is for the human is a key aspect of this exploration. For instance, characterising nature in atomistic terms, awaiting discovery by scientific investigation, posits science as an exteriorised epistemic exercise. Considering Merleau-Ponty's flesh however, the potential emerges for an identification of the ontologically productive, rather than observationally passive, capacities of scientific practice. This will take us beyond mere representationalism, in which scientific practice is understood to be a set of theories derived from second-degree representations of the world. For Merleau-Ponty, in the act of perceiving a table, it is not that there is one table in the world and one in the perceiver's mind, but instead a singular fleshed phenomenon of table. Similarly, for physicist Niels Bohr, theorising should be an "embodied practice," rather than "a spectator sport of matching linguistic representations to preexisting things” (Barad, 2007, p. 54).

Wanting to avoid the realm of representationalism, and its associate, "reflection," physicist Karen Barad prioritises the importance of quantum theories of diffraction. Diffractive processes are more concerned with entanglements than autonomies. Rather than uncovering a world of separate facts-in-themselves, diffractions are attuned to the "differences that our knowledge- 
making practices make and the effects they have on the world” (2007, p. 72). In order to comprehend one's involvement in, rather than mere observation of, the world and its production, a clarification of the core concepts within quantum mechanics is necessary.

\section{Quantum Foundations: The Wave and Particle Paradox}

Diffractions can be understood as the resultant effect when waves overlap and consequently interfere with each other. For example, if two stones are dropped into the same pond within a certain period of time, the ripple effects from each will interfere with the other, producing diffraction/interference patterns of increased amplitudes and troughs where the equivalent points of each wave meet. When this happens, their amplitudes combine and the resultant formation is a composite waveform. ${ }^{2}$ This behaviour of waves is in contrast to that of matter as particles, which by occupying a point in space, at a particular point in time, are not able to overlap with each other. Classical physics typically considers matter as waveform and matter as particle to be mutually exclusive. That is, an atom, as the building block of physical reality, cannot exhibit both wave behaviour and particle behaviour. A scientific exercise, which invokes, and yet problematises, this mutual exclusivity of the wave/particle pairing is found in what is known as a two-slit diffraction experiment. This procedure shoots matter at a board which features two equally sized cut slits. The matter should either leave a record of which slit it passed through, indicating particle behaviour, or otherwise be observable as an interference pattern on a final screen, exemplifying wave behaviour that has passed through both slits.

It is with such a method that something like the nature of light is explored. This is illustrated in debates between physicists during the early twentieth century, when experiments being undertaken in the field of atomic physics suggested that under certain experimental conditions, light manifests particle characteristics, whereas under other experimental configurations it manifests with wavelike characteristics. Because of this, physicists Niels Bohr and Albert Einstein employed two-slit thought (gedanken) experiments in order to examine the increasingly paradoxical nature of wave and particle behaviour. When this experiment is undertaken using individual electrons, each electron should pass through either slit, one at a time, whereby the possibility of interaction with another electron has been removed. Thus by being deployed individually, and by only passing through one slit in the experimental apparatus, each should exhibit particle behaviour.

Remarkably, despite this method of individual discharge, experiments such as those conducted by physicists Thomas Young and Augustine Fresnel illustrate that the electrons, tiny particles of matter, produce a diffraction pattern! ${ }^{3}$ Because the only possible mode of interference in such a case could be if the electron passes through both slits simultaneously, like a wave, and thus interferes with itself, information about which-slit each passes through is required in order to confirm the particle or wave status of each electron. Einstein and Bohr discussed a modification of the apparatus in order to perform such an experiment. ${ }^{4}$ However this was at a time before scientific technology allowed it to be possible. This would replace the fixed base of the two-slitted diaphragm with a suspension installed on a spring, allowing a detection of which-slit the electron had passed through by recording whether a displacement of the diaphragm had occurred. 


\section{Can we Remove the Observer from the Observed?}

What is now an issue is the displacement disturbance between the movable apparatus and the electron. Einstein believed that this disturbance could be calculated and subtracted out from the electron's recorded properties. ${ }^{5}$ Consistent with his rejection of the interpretation that positions in space-time could never be completely known, this would facilitate an identification of the position and momentum qualities of the electron, independent of the interaction from the measuring process. That is, independent of the effects of its pass through the two-slit apparatus. But can we say this? Merleau-Ponty has already presented us with a philosophy that posits an irreducible implication between observer and observed. Can scientific practice refute this?

In order to address this question, let's examine a typical measuring process. A flash camera is mounted on a plate in a dark room. The position of the object being measured can only be determined if the photographic plate is fixed. This is because, as Barad remarks, "if we were to allow the plate to move during the measurement, we would not have a viable way of defining the particle's position" (2007, p. 111). Alternatively, when attempting to define momentum, it is necessary that the camera rests on a movable platform rather than a fixed one. The reason for this is that in measuring momentum, it must also be possible to measure to what extent the photographic platform moves back as a result of its interaction with the object being measured. Here we are reminded of Merleau-Ponty's argument that for the embodied subject, to see is to possess a body that is capable of being seen. Likewise, in experimentation, in order to see the position of the particle, the apparatus-as-see-er must have a see-able position for the particle. Correlatively, to be able to observe an object's momentum, the momentum of the apparatus-asobserver must be visible to the object-as-observed.

The ramification of having such mutually exclusive experimental configurations is that it is not possible to simultaneously determine the position and the momentum of a "thing." As Bohr states, each measurement "demands mutually exclusive experimental arrangements" (1963b, p. 41). There is a complementary relationship between the use of an experimental apparatus which determines: (i) which-slit information (that is, which path the particle travels through), and; (ii) the materialisation of an interference/wave pattern on the final detecting screen. Consequently, "we are presented here with a choice of either tracing the path of a particle or observing interference effects" (1963b, p. 41). That is, one cannot concurrently obtain which-slit/particle information and an interference/wave pattern, given that each measurement requires a different experimental configuration, a configuration that will already be entangled in the production of either the particle or wave properties of the matter under investigation.

The constitution of the experimental apparatus will determine which properties become determinate. It is only via the configuration of the measuring apparatus that the object comes to have particle or wave behaviour at all. The notion of a measurement-independent object is negated by this indeterminacy, meaning that the effect of the measurer in the object being measured is unable to be distinguished from the object itself. Thus Bohr disagrees with Einstein. Such a conclusion affirms Merleau-Ponty's problematisation of the subject/object divide. Just as for Merleau-Ponty it is only via the act of perception that subject and object manifest, similarly in wave/particle experimentation the observed does not exist with pre-given properties prior to its being "produced" as the object that it is by an implicated observer. The boundary between the observer and the observed is indeterminate due to the conditional nature of the physical apparatus that is required to bring about a measurement of the observed at all. 


\section{What can be Determined (An Ontology), Not What can be Known (An Epistemology)}

In considering this production of Being for the subject and the object, for the observer and the observed, in this act that Merleau-Ponty calls perception, and which Bohr calls measurement, it is useful to distinguish what Bohr describes as indeterminacy, from Heisenberg's "uncertainty principle." In his famous article, the $20^{\text {th }}$ century German physicist Werner Heisenberg considers the impact upon an electron when it is measured using a high-energy microscope. Heisenberg's interpretation is that there is an interaction between the electron, and the photon emitted from the flash of the microscope, in the process of measuring the electron. As a result of this interaction, we cannot know both its momentum and position simultaneously due to the disturbance caused by the measuring process. For Heisenberg, this is not a matter of Bohrian indeterminacy, but rather a disturbance, which prevents us knowing the measurement-independent values of the thing itself. Heisenberg duly believes that the uncertainty relation is an epistemic principle, limiting what we can know about an object under investigation, whereby "an accurate measurement of one observable quantity necessarily produces uncertainties in one's knowledge of another" (Heisenberg, 1930, p. 20). The consequence of this is that it posits a determinate value for the electron that is assumed to exist independently of the experiment. As we have seen however, perception for Merleau-Ponty is not an act providing access to a pre-existing object for a distinct subject. Equally for Bohr, the properties of an object do not even come to manifest without a particular apparatus that is configured to measure for them.

For Bohr, complementary values such as position and momentum are not simultaneously measurable, in that it is not possible to determine "both the momentum and the position of an object" (Bohr, 1963b, p. 40). It is not a matter of an unknowability due to the disturbance of a pre-existent, but rather an impossibility in terms of what can be in existence simultaneously. The notion of disturbance is irrelevant because the corresponding properties are not determinate without the entangled involvement of a particular measuring apparatus. There are simply no preexisting qualities to disturb. Thus, it is not that the observed interference/wave qualities of the electrons are disrupted by the attempted determination of which-slit/particle information. It is not that wave or particle behaviours exist as pre-given properties of an object, and that the measuring process discovers some of these properties and destroys others. Rather, wave and particle behaviour, properties which characterise an object, only come into being through the experiment itself. This characterises the specificity of complementary experimental configurations. These are the grounds upon which Bohr objects to the Heisenberg evaluation that a disturbance of the preexisting properties is the issue in the process of measurement, and that this functions as a limit to the knowledge we can have.

With this in mind, in the early 1990's, a team of physicists led by Marlan Scully employed recent developments in quantum optics to examine Heisenberg's uncertainty claims. In these experiments, two collimated beams of atoms are streamed towards two micromaser cavities aligned with the two-slits in the diffraction grating as per previous examples. As Barad describes for us, before the atoms hit the cavities:

a laser beam is used to excite the atoms to a higher energy state ... which then emit a photon into the cavity, thereby leaving a physical trace that marks which-path information. The de-excited atoms then pass out of the cavity, go 
through the two-slit diffraction grating, and are detected on a fixed screen. (2007, p. 306)

The important point to take from this process is that Scully's team excites and de-excites the atoms without disturbing their centre-of-mass motion. That is, they obtain which-path or particlelike information without introducing large uncontrolled phase factors (disturbances) to the interfering beams (Scully et. al, 1991, p. 111).

In being able to track which-path information without causing any disturbance to the motion of the atom, it is duly concluded that the Heisenberg uncertainty principle, by positing a disturbance to the pre-measured properties of the atom, does not explain the effect of the measuring apparatus on the atom. Despite the lack of disturbance, the team notes a Bohrian complementarity between the availability of which-path information and an interference pattern. In short, they can have one or the other, but not both simultaneously. Scully's team describes this as a correlation "between the measuring apparatus and the systems being observed," that is, the entanglement of the "object of observation" and the "agencies of observation." Just as MerleauPonty argues that perception enacts the blurring of the very fabric which constitutes the individual-as-subject and the world-as-object, similarly Bohrian analysis has been validated by positing an implication, rather than a separation, of the observer and the observed. Consequently, via the act of measurement, "we are faced with the impossibility of drawing any sharp separation between an independent behaviour of atomic objects and their interaction with the measuring instruments" (Bohr, 1963b, pp. 46-47). In being unable to draw a "sharp separation” between objects and the measuring instruments, it is as if neither actually exists without the interaction which brings about the determinable cut between them, whereby they concurrently manifest as objects being measured and instruments doing the measuring.

\section{A "Fleshing Out" of the "Flesh of the World"}

Here Merleau-Ponty's interpretation of the incarnation of subject and object in the act of perception is evoked. Perception, as the self-differentiation of the "flesh of the world," manifests via a body that is located at a proximal distance from a world that it is. It is only because of this distance that perception is possible, producing a determinable subject and object, a determinacy as distinguishable as the sharp separation in the Bohrian cut. Subject and object for MerleauPonty, as fleshed incarnations, whilst never independent of the other, are never self-identical either. If the flesh were to entirely coincide with itself, perception would not be possible. This is because there would be an undifferentiated self-presence, meaning that subject and object would not manifest. Subject and object are mutually exclusive, but inseparable. This mimics the implicated, but cut-separated, relation of agencies-of-observation to objects-of-observation in Bohrian experimentation. As we will observe however, this congruence between MerleauPontian perception and Bohrian measurement should be comprehended as intimately parallel, rather than straightforwardly identical.

The lack of a pre-given, or inherent, distinction between the objects under observation and the agent of observation is the foundation of Bohr's conception of quantum wholeness. Here Merleau-Ponty correlatively argues that in the act of perception the subject is implicated in the object it perceives, in the manner with which the gaze clothes an object with its own flesh in order to be able to see it. This is not a chronological statement, in terms of positing an object 
before-and-after being “clothed," but rather a logical one. If the object being perceived was not of the same matter as the perceiver, if they were not incarnated, via the same Bohrian cut, then subject and object would not manifest at a proximal distance from one another, and instead there would be indeterminacy. Accordingly, Merleau-Ponty notes that "we could not dream of seeing things 'all naked' because the gaze itself envelops them, clothes them with its own flesh" (1968, p. 131). This perpetual entanglement is why, for Bohr, proper quantum phenomena must describe all features of the experimental configuration. It is within the specificity of a largerphenomenon-in-flux that the Bohrian cut distinguishes components like observer and observed, and that Merleau-Ponty witnesses the subject/object emergence. Accordingly, Bohr advocates "the application of the word phenomenon exclusively to refer to the observations obtained under specified circumstances, including an account of the whole experimental arrangement" (2007 [orig. 1948], p. 312). It is more than mere coincidence that Bohr's application of the umbrella term "phenomena," to describe measurement in a manner beyond a straight-forward observation, coincides with Merleau-Ponty’s “phenomenology of perception.” Considering this synchronicity, it is surprising then that each has a contrasting appreciation of the role of human corporeality within such phenomena. Moreover, it is via such an insight that a significant difference between Merleau-Pontian perception and Bohrian measurement will emerge.

\section{The Destabilised Human}

Just as the object does not materialise before the cut of the experimental apparatus that makes it determinate, similarly the human, as implicated in the operation of measuring instruments, should not be inherently privileged as an anteriority either. As Barad states, boundary-making practices are "not a human-dependent characteristic but a feature of the world in its differential becoming. The world articulates itself differently” (2007, p. 149). In evoking notions of the perpetual becoming of a global flesh, Merleau-Ponty would endorse this appraisal, especially given that it does not impose a human/subject-centrism. The perpetual self-differentiation of Merleau-Ponty's flesh incarnates human corporeality as a thing amongst/within other things after all. But does Bohr implicate, and thus destabilise, the human in an equivalent manner? Furthermore, if he does not, is he even required to?

In one sense, Bohr does declare an appreciation for the ambiguity of bodily boundaries. For instance, there is his description of how:

when a stick is held loosely, it appears to the sense of touch to be an object. When, however, it is held firmly, we lose the sensation that it is a foreign body, and the impression of touch becomes immediately localised at the point where the stick is touching the body under investigation. (Bohr, 1963a, p. 99)

In further evidencing the correlation with Merleau-Ponty's conception of the limitless human body, we can observe that the phenomenologist employs almost this exact example himself: "the blind man's stick has ceased to be an object for him, and is no longer perceived for itself; its point has become an area of sensitivity" whereby, as illustrated earlier, "to get used to a hat, a car or a stick is to be transplanted into them, or conversely, to incorporate them into the bulk of our own body” (1962, p. 143). ${ }^{6}$ Clearly, bodily boundaries are as ambiguous for Bohr as they are for Merleau-Ponty, and indeed as for Schilder, as earlier observed. However, despite this conceptual 
consistency, the human body is disregarded in terms of the experimental apparatus by Bohr, instead seemingly being stationed as an outside observer/organiser. What we will now need to determine, in considering the congruence between Merleau-Pontian perception and Bohrian measurement, is how such a difference manifests, and what the ramifications are for the ontological equivalence of phenomenological perception to quantum mechanical measurement.

The interpretation that Bohr externalises the human is based on the assumption that the apparatus is chosen by an experimenter who then records the results from a position of neutrality. Despite Bohr's demand that apparatuses are not passive observing instruments, and even considering his associated argument which endorses the lack of an inherent distinction between the object being measured and the instrument of measurement, the human still appears to be the limit of the Bohrian apparatus. Physicist Percy Bridgman notes in this regard that even quantum knowledge requires some kind of "knower," and for Bohrian quantum theory, the human "knower is not part of the picture, but he stands on the outside looking on" (1958, p. 178). Merleau-Ponty de-limits the boundary of human corporeality in order to argue that there is nothing outside of "body." To the contrary for Bohr, an apparatus, as the thing, which conditions the possibility of determinate subject and object boundaries, is inherently limited in the manner with which it is sealed off from outside, operational influences. Thus, the human is exteriorised from the apparatus and it's functioning, which ignores the fact that most experiments are not always initially successful. An experimental apparatus is just that, "experimental,” and typically requires constant human monitoring, interference and involvement.

\section{The Phenomenal, Corporeal, Experimental Apparatus}

This issue of the human, corporeal implication in the scientific apparatus is fascinatingly illustrated in what is known as the Stern-Gerlach experiment. Conducted in 1922, this experiment was concerned with understanding space quantisation as a real phenomenon. As physicist Hans Reichenbach describes, it involved "passing a collimated beam of electrons through a non-uniform magnetic field and recording the pattern of electron impacts on a sensitised screen located downstream” (1979, p. 581). In order to undertake this experiment, physicist Walther Gerlach was employed as an assistant to Otto Stern, who was working on atomic beam experiments in an adjacent building. After having performed the experiment, the sensitised detector screen was removed by Stern, however no trace of the silver atom beam was evident. Then, as the disappointed physicists inspected the glass plate more closely, a trace began to emerge. Stern, explaining why this occurred, notes the influence of his cigar stained breath in the functioning of the experimental apparatus:

I was then the equivalent of an assistant professor. My salary was too low to afford good cigars, so I smoked bad cigars. These had a lot of sulphur in them, so my breath on the plate turned the silver into silver sulphide, which is jet black, so easily visible. It was like developing a photographic film. ${ }^{7}$

Stern's tobacco habit and financial position had thus played a productive role in the experiment. The embodied scientist, far from being removed from the apparatus, was corporeally implicated in not only the measuring apparatus, but also in the object being measured! Clearly such an example works against the Bohrian assumption that an outside boundary to the apparatus exists 
at the point where the laboratory equipment finishes. Reality does not feature a neat boundary between the social realm and the scientific realm, but rather each constitutes the other, whereby the ontology of each becomes determinate as a result of their implicated practice. Indeed, the culture/nature distinction is once again shown to be dismantled in a fashion that is consistent with the all-incarnating activity of Merleau-Ponty's flesh.

The implication of the human in the instruments of the apparatus, and in the products of experimentation, means that, consistent with Merleau-Ponty's interpretation, all bodies are produced beyond the apparent corporeal limits of the subject or of the apparatus. Is it problematic, or indeed contradictory, for Bohr to neglect the boundary that is assumed between the apparatus and its human orchestrator that manifests in the laboratory configuration? Bohr insists, after all, that an objective, "unambiguous account of proper quantum phenomena must, in principle, include a description of all relevant features of the experimental arrangement" (1963c, p. 4). This question is particularly relevant considering that his quantum argument generally argues that if an object is not part of a larger physical arrangement, then it will not have determinate boundaries, and thus not be objectively distinguishable. Quantum physicist Andrew Whitaker similarly observes this character of Bohrian physics, noting that it is an "essential part of Bohr's analysis that measuring apparatus must be of macroscopic dimensions - one might say, as large as possible” (2006, p. 172). Without a larger, phenomenal frame of reference, the "outside" of the apparatus is as indeterminate as the internal boundaries of subject/object, or observer/observed, would be were it not for the marking off that is enacted by the apparatus.

This exteriorisation of the human is the only major difference between the ontologies of Bohr's apparatus and Merleau-Ponty's perception; nevertheless it is a considerable one. Earlier it was indicated that we would need to address how such a difference manifests between these two seemingly congruent mechanisms, and indeed, what the ramifications are for the potential of an ontological equivalence between phenomenological perception and quantum mechanical measurement. What is becoming obvious is that Merleau-Pontian perception, and Bohrian measurement, act in an intimately parallel manner without being straightforwardly identical.

Phenomenological perception, as the originary becoming of the flesh-of-the-world, incarnates as a most primordial ontology. Quantum mechanical measurement is also an incarnating, rather than merely a representing, process. Whilst quantum mechanics is not restricted to the micro realm, its laboratory manifestation envisages the physical reality that it produces at a micro level (atoms as waves or particles). This requires a certain idealisation that is integral to the methodology of common scientific endeavour, whereby Bohr might justify positioning the human in a way that they are distinguished by their orchestration of such idealisation. Interestingly, if to be distinguished is to be structurally produced, the human would not be exteriorised from the apparatus by which they are distinguished-produced whatsoever.

Quantum mechanics could thus be conceptually, primordially identical to phenomenological perception, however only if the laboratory based quantum measurement with which we have been concerned is seen to emerge as a particular incarnation, or version of it. An incarnation which incarnates. This is not cutting the laboratory off from the rest of reality. Rather, it is identifying what occurs in the laboratory as a specific manifestation of a primordial quantum becoming, proximally distanced from such primordiality in order that it becomes, like all things, distinguishably. This could engender the more sympathetic reading that, likewise, the Bohrian human is distinguished, rather than excluded, from the experimental apparatus. 
According to this logic, Merleau-Pontian perception and Bohrian measurement have contrasting ontological states. Quantum measurement does what phenomenological, fleshed perception does, only because quantum measurement functions as an expression of this primordial flesh-of-the-world. In perceiving itself via the concurrent measurement-manifestation of agents of observation and objects of observation, the flesh proximally distances itself from itself. Measurement, as a perceptive-productive process of the flesh, cannot entirely coincide with the flesh which it is. This proximal differentiation of flesh-from-flesh marks quantum measurement as a phenomenological movement, sharing the same ontologically productive mechanism as perception, however as just illustrated, not the same basic ontological status.

\section{A Reiteration in Conclusion}

To reiterate, Bohr, in blurring the line between the measuring apparatus and the object being measured, dismantles the distinction between observer and observed. This functions similarly to the manner with which Merleau-Ponty problematises the distinction between perceiving subject and perceived object. For Bohr and Merleau-Ponty, there is not a pre-given observer and observed, or an inherent subject and object, but rather all only come into being/determinacy via the incarnating, rather than the observing, capacities of Bohrian-measurement/Merleau-Pontianperception. As we have seen, there is a divergence in terms of the role of human corporeality for each. This, however, is due to their contrasting ontological statuses, rather than evidencing any difference in their respective ontological mechanisms. Both, in this regard, are theories of ontological production, rather than of the epistemic discovery of a pre-existent. Thus, in addressing the question of the article as initially presented, both Merleau-Ponty's phenomenology of perception, and Bohr's quantum measurement, demand that we cannot reduce Being to a separation of knower from what can be known, or of observer from what can be observed.

\section{Endnotes}

${ }^{1}$ In terms of the central tenet of this article, which will argue that there is congruence between Merleau-Ponty's characterisation of perception and Bohr's understanding of quantum experimental measurement, this notion that a thing can only see when it is capable of being seen will emerge as a crucial link.

2 Physicists Larry Kirkpatrick and Gerald Wheeler provide a useful description of composite waveform production in Physics: A world view (2006, p. 317).

${ }^{3}$ In this regard, a renowned "treatise on light" is proffered by physicists Christiaan Huygens, Thomas Young, and Augustin Fresnel in The wave theory of light: Memoirs of Huygens, Young and Fresnel (2008).

${ }^{4}$ For an account of this experimental development, see physicist Andrew Whitaker's Einstein, Bohr and the quantum dilemma: from quantum theory to quantum information (2006). Physicists Jagdish Mehra \& Helmut Rechenberg also track Einstein and Bohr's interactions in this regard in The historical development of quantum theory: volume 6, part 1 (2000). 
${ }^{5}$ This is consistent with the position of Newtonian physics, something Karan Barad summaries in Meeting the universe halfway (2007, pp. 107-109). See also Mehra \& Rechenberg (2000).

${ }^{6}$ It is important to note that I do not interpret this as an endorsement of "post-humanism” from Merleau-Ponty. There are two possible readings for why this is the case. The first is that the stick does not "artificially extend" the human, given that the human, the stick, and all other things are incarnates of the flesh-of-the-world. However, all incarnates are not ontologically equal, whereby this proximal distance/co-implication between human and stick is brought about by human purposes. That is, the intentions of the human are served by the stick in a form of expansion or absorption.

The second reading argues that it is not that the intentions of the human are served by the stick. The relation between the human and the stick is asymmetrical, however this is an asymmetry which is arranged organisationally, in order to distinguish things from other things, rather than hierarchically, in order to privilege things over other things. The stick-as-flesh only emerges distinguishably as a stick due to its proximal differentiation from the human-as-flesh which emerges concurrently. The stick-as-flesh is thus so implicated in the production of the human-as-flesh that its humanness is undeniable. The ontological privilege in this scenario is with the flesh-of-the-world, from which the human, and the stick, manifest.

7 This citation is taken from physicists Bretislav Friedrich and Dudley Herschbach's Space Quantization: Otto Stern's Lucky Star (1998, pp. 178-79), in which the Stern-Gerlach experiment is heralded as "a treasured episode from the heroic age of atomic physics... elegantly simple in its conception, extraordinarily startling in its outcome, and extremely fruitful in its legacy” (p. 165). 


\section{References}

Barad, K. (2007). Meeting the universe halfway: Quantum physics and the entanglement of matter and meaning. Durham, NC: Duke University Press.

Bohr, N. (1963a). The philosophical writings of Niels Bohr, vol. 1: Atomic theory and the description of nature. Woodbridge, CT: Ox Bow Press.

Bohr, N. (1963b). The philosophical writings of Niels Bohr, vol. 2.: Essays, 1933 - 1957, on Atomic Physics and Human Knowledge. Woodbridge, CT: Ox Bow Press.

Bohr, N. (1963c). The philosophical writings of Niels Bohr, vol. 3. Essays, 1958 - 1962, on Atomic Physics and Human Knowledge. Woodbridge, CT: Ox Bow Press.

Bohr, N. (2007 [orig. 1948]). On the notions of causality and complementarity. Dialectica, 2 (34), 312. doi: 10.1111/j.1746-8361.1948.tb00703.

Bridgman, P. (1958). Remarks on Niels Bohr's talk. Daedalus, 87(2), 175-177. Retrieved from http://www.jstor.org/stable/20026445.

Cooney, B. (2000). The place of mind. Belmont, CA: Wadsworth Thomson Learning. Descartes, R. (1999 [orig. 1643]). Correspondence with Elizabeth. In G. M. Ross (Trans.), Descartes: correspondence with Elizabeth. Retrieved from http://www.philosophyleeds.ac.uk/GMR/hmp/texts/modern/descartes/elizabeth/elizindex. $\underline{\mathrm{html}}$.

Friedrich, B. \& Herschbach, D. (1998). Space quantization: Otto Stern’s lucky star. Daedalus, 127, 165-191.

Grosz, E. (1994). Volatile bodies: Toward a corporeal feminism. Sydney, NSW: Allen \& Unwin.

Heisenberg, W. (1930). The physical principles of quantum theory (C. Eckart \& F. Hoyt, Trans.). New York: Dover Publications.

Hetherington, S. (2003). Reality? knowledge? philosophy! Edinburgh, Midlothian: Edinburgh University Press.

Huygens, C., Young, T., Fresnel, A., \& Arago, F. (2008 [orig. 1900]). The wave theory of light: Memoirs of Huygens, Young and Fresnel. H. Crew (Ed.), Knoxville, TN: American Book Company.

Kirkpatrick, L., \& Wheeler, G. (2006). Physics: A world view. Melbourne, VIC: Cengage Learning.

Mehra, J. \& Rechenberg, H. (2000). The historical development of quantum theory: volume 6, part 1. New York, NY: Springer. 
Merleau-Ponty, M. (1962). Phenomenology of perception (C. Smith Trans.). London and New York, NY: Routledge.

Merleau-Ponty, M. (1968). The visible and the invisible (A. Lingis Trans.). Evanston, IL: Northwestern University Press.

Reichenbach, H. \& Salmon, W. (1979). Hans Reichenbach, logical empiricist, New York, NY: Springer Science \& Business.

Schilder, P. (1950). The image and appearance of the human body. New York, NY: International Universities Press Inc.

Scully, M., Berthold-Georg E., \& Walther, H. (1991). Quantum optical tests of complementarity. Nature, 351, 111-116.

Whitaker, A. (2006). Einstein, Bohr and the quantum dilemma: From quantum theory to quantum information, Cambridge: Cambridge University Press. 